\title{
Engel-like conditions in fixed points of automorphisms of profinite groups
}

\author{
Cristina Acciarri ${ }^{1}$ (D) Danilo Silveira ${ }^{2}$
}

Received: 5 February 2019 / Accepted: 3 June 2019 / Published online: 10 June 2019

(c) Fondazione Annali di Matematica Pura ed Applicata and Springer-Verlag GmbH Germany, part of Springer Nature 2019

\begin{abstract}
Let $q$ be a prime and $A$ an elementary abelian $q$-group acting as a coprime group of automorphisms on a profinite group $G$. We show that if $A$ is of order $q^{2}$ and some power of each element in $C_{G}(a)$ is Engel in $G$ for any $a \in A^{\#}$, then $G$ is locally virtually nilpotent. Assuming that $A$ is of order $q^{3}$, we prove that if some power of each element in $C_{G}(a)$ is Engel in $C_{G}(a)$ for any $a \in A^{\#}$, then $G$ is locally virtually nilpotent. Some analogues of quantitative nature for finite groups are also obtained.
\end{abstract}

Keywords Profinite groups · Automorphisms · Centralizers · Engel-like conditions

Mathematics Subject Classification Primary 20E18, 20E36 - Secondary 20F45, 20F40, 20D45, 20F19

\section{Introduction}

A profinite group is a topological group that is isomorphic to an inverse limit of finite groups. In the context of profinite groups, all the usual concepts of group theory are interpreted topologically. In particular, by a subgroup of a profinite group we always mean a closed subgroup and a subgroup is said to be generated by a set $S$ if it is topologically generated by $S$. See, for example, [24] for these and other properties of profinite groups. Many remarkable

Dedicated to Pavel Shumyatsky on the occasion of his 60th birthday.

Cristina Acciarri was supported by the Conselho Nacional de Desenvolvimento Científico e Tecnológico $(\mathrm{CNPq})$ and Fundação de Apoio à Pesquisa do Distrito Federal (FAPDF)-Brazil, and Danilo Silveira by the Coordenação de Aperfeiçoamento de Pessoal de Nível Superior (CAPES)- Brazil.

Cristina Acciarri

acciarricristina@yahoo.it

Danilo Silveira

sancaodanilo@ufg.br

1 Department of Mathematics, University of Brasilia, Brasília-DF 70910-900, Brazil

2 Department of Mathematics, Federal University of Goiás, Catalão, GO 75704-020, Brazil 
results on profinite groups were deduced using the Lie-theoretical machinery developed for the solution of the restricted Burnside problem [26,27,29]. For instance, using Wilson's reduction theorem [23], Zelmanov proved that a profinite group is locally finite if and only if it is torsion [28]. Recall that a group $G$ is said to have a certain property locally if any finitely generated subgroup of $G$ possesses that property. We say that a group $G$ is torsion if all of its elements have finite order.

Another well-known result of Wilson and Zelmanov [25, Theorem 5] tells us that a profinite group is locally nilpotent if and only if it is Engel. If $x, y$ are elements of a (possibly infinite) group $G$, the commutators $[x, n y]$ are defined inductively by the rule

$$
[x, 0 y]=x, \quad[x, n y]=[[x, n-1 y], y] \text { for all } n \geq 1 .
$$

Recall that an element $x$ is called a (left) Engel element if for any $g \in G$ there exists $n$, depending on $x$ and $g$, such that $\left[g,{ }_{n} x\right]=1$. A group $G$ is called Engel if all elements of $G$ are Engel. The element $x$ is called a (left) $n$-Engel element if for any $g \in G$ we have $[g, n]=1$. The group $G$ is $n$-Engel if all elements of $G$ are $n$-Engel.

Later on, in [4], Bastos and Shumyatsky considered profinite groups with Engel-like conditions. They showed in [4, Theorem 1.1] that if $G$ is a profinite group in which for every element $x \in G$ there exists a natural number $q=q(x)$ such that $x^{q}$ is Engel, then $G$ is locally virtually nilpotent. We recall that a profinite group posses a certain property virtually if it has an open subgroup with that property. Note that the previous result can be viewed as a common generalization of both the Wilson-Zelmanov results on profinite groups stated above.

By an automorphism of a profinite group, we mean a continuous automorphism. We say that a group $A$ acts on a profinite group $G$ coprimely if $A$ has finite order, while $G$ is an inverse limit of finite groups whose orders are relatively prime to the order of $A$. In the literature, there are many well-known results showing that if $A$ is a finite group acting on a finite group $G$ in such a manner that $(|A|,|G|)=1$, then the structure of the centralizer $C_{G}(A)$ (the fixed-point subgroup) of $A$ has a strong influence over the structure of $G$ (see, for instance, $[2,10,21,22])$. A similar phenomenon holds in the realm of profinite groups: We see that imposing restrictions on centralizers of coprime automorphisms result in very specific structures for the group $G$. Given an automorphism $a$ of a profinite group $G$, we denote by $C_{G}(a)$ the centralizer of $a$ in $G$, that is, the subgroup formed by the elements fixed under $a$. In particular, the following theorems were established in [20, Theorem 1.1] and [2, Theorem B2], respectively.

Theorem 1 Let $q$ be a prime and A an elementary abelian q-group of order at least $q^{2}$ acting coprimely on a profinite group $G$. Assume that the centralizer $C_{G}(a)$ is torsion for each $a \in A^{\#}$. Then $G$ is locally finite.

Theorem 2 Let $q$ be a prime and A be an elementary abelian q-group of order at least $q^{2}$ acting coprimely on a profinite group $G$. Assume that all elements in $C_{G}(a)$ are Engel in $G$ for each $a \in A^{\#}$. Then $G$ is locally nilpotent.

Here and throughout the paper, $A^{\#}$ denotes the set of nontrivial elements of $A$. The proofs of the above results involve a number of deep ideas. In particular, Lie-theoretical results of Zelmanov [26,27,29] obtained in his solution of the restricted Burnside problem are combined with a criteria for a pro- $p$ group to be $p$-adic analytic in terms of the associated Lie algebra due to Lazard [12], and a theorem of Bahturin and Zaicev [3] on Lie algebras admitting a soluble group of automorphisms whose fixed-point subalgebra satisfies a polynomial identity. Moreover, Theorems 1 and 2 rely heavily on Zelmanov's theorem about local finiteness of 
torsion profinite groups and on the Wilson-Zelmanov result on local nilpotency of Engel profinite groups, respectively.

In the present paper, we consider profinite groups admitting an action by an elementary abelian group under which the centralizers of automorphisms satisfy the property that some power of any element is Engel. In this context of profinite groups, by some power of any element in the centralizers we mean that, for every element $x$ in the centralizers, there exists a natural number $n=n(x)$ such that $x^{n}$ is an Engel element. Our first goal is to establish the following result.

Theorem 3 Let $q$ be a prime and A an elementary abelian group of order $q^{2}$. Suppose that $A$ acts coprimely on a profinite group $G$ and assume that some power of each element in $C_{G}(a)$ is Engel in $G$ for any $a \in A^{\#}$. Then $G$ is locally virtually nilpotent.

Using Theorem 3 in combination with the positive solution of the restricted Burnside problem $[26,27,29]$, the following quantitative result for finite groups can be obtained.

Corollary 1 Let $m, d$ be integers, $q$ a prime and $A$ an elementary abelian group of order $q^{2}$. Suppose that $A$ acts coprimely on a $m$-generated finite group $G$ and assume that all $d t h$ powers of elements in $C_{G}(a)$ are $n$-Engel in $G$ for each $a \in A^{\#}$. Then there exist positive integers $e$ and $c$, depending only on $m, n, q$ and $d$, such that $G$ has a normal subgroup $N$ with nilpotency class at most $c$ and $|G / N|$ is at most $e$.

If, in Theorem 2, we relax the hypothesis that every element of $C_{G}(a)$ is Engel in $G$ and require instead that every element of $C_{G}(a)$ is Engel in $C_{G}(a)$, we see that the result is no longer true. Indeed, an example of a finite nonnilpotent group $G$ admitting an action of a noncyclic group $A$ of order four such that $C_{G}(a)$ is abelian for each $a \in A^{\#}$ can be found for instance in [1]. On the other hand, in [1], the authors proved that if $A$ is an elementary abelian $q$-group of order at least $q^{3}$, with $q$ a prime, acting coprimely on a profinite group $G$ in such a manner that $C_{G}(a)$ is locally nilpotent for each $a \in A^{\#}$, then $G$ is locally nilpotent. Another purpose of the present paper is to establish the following related result.

Theorem 4 Let $q$ be a prime and A an elementary abelian group of order $q^{3}$. Suppose that $A$ acts coprimely on a profinite group $G$ and assume that some power of each element in $C_{G}(a)$ is Engel in $C_{G}(a)$ for any $a \in A^{\#}$. Then $G$ is locally virtually nilpotent.

Our next result is an analogue of Corollary 1.

Corollary 2 Let $m, d$ be integers, $q$ a prime and A an elementary abelian group of order $q^{3}$. Suppose that $A$ acts coprimely on a $m$-generated finite group $G$ and assume that all $d$ th powers of elements in $C_{G}(a)$ are $n$-Engel in $C_{G}(a)$ for each $a \in A^{\#}$. Then there exist positive integers $e$ and $c$, depending only on $m, n, q$ and $d$, such that $G$ has a normal subgroup $N$ with nilpotency class at most $c$ and $|G / N|$ is at most $e$.

The paper is organized as follows. In Sect. 2, we present the Lie-theoretical machinery that will be useful within the proofs. Section 3 is devoted to proving Theorem 3 and Corollary 1 , and in the last section, we establish Theorem 4 and Corollary 2.

The notation is standard. Throughout the paper, we use, without special references, the following well-known properties of coprime actions (see, for example, [18, Lemma 3.2]).

If $\alpha$ is a coprime automorphism of a profinite group $G$, then $C_{G / N}(\alpha)=C_{G}(\alpha) N / N$ for any $\alpha$-invariant normal subgroup $N$.

If $A$ is a noncyclic elementary abelian group acting coprimely on a profinite group $G$, then $G$ is generated by the subgroups $C_{G}(B)$, where $A / B$ is cyclic. 


\section{Associated Lie algebras}

Let $L$ be a Lie algebra over a field $K$ and $X$ a subset of $L$. By a commutator in elements of $X$, we mean any element of $L$ that can be obtained as a Lie product of elements of $X$ with some system of brackets. If $x_{1}, \ldots, x_{k}, x, y$ are elements of $L$, we define inductively

$$
\left[x_{1}\right]=x_{1} ;\left[x_{1}, \ldots, x_{k}\right]=\left[\left[x_{1}, \ldots, x_{k-1}\right], x_{k}\right]
$$

and $[x, 0 y]=x ;[x, m y]=\left[\left[x,_{m-1} y\right], y\right]$, for all positive integers $k, m$. As usual, we say that an element $a \in L$ is ad-nilpotent if there exists a positive integer $n$ such that $\left[x,{ }_{n} a\right]=0$ for all $x \in L$. If $n$ is the least integer with the above property, then we say that $a$ is ad-nilpotent of index $n$. Denote by $F$ the free Lie algebra over $K$ on countably many free generators $x_{1}, x_{2}, \ldots$. Let $f=f\left(x_{1}, x_{2}, \ldots, x_{n}\right)$ be a nonzero element of $F$. The algebra $L$ is said to satisfy the identity $f=0$ if $f\left(l_{1}, l_{2}, \ldots, l_{n}\right)=0$ for any $l_{1}, l_{2}, \ldots, l_{n} \in L$. In this case, we say also that $L$ satisfies a PI (polynomial identity) or that $L$ is a PI-algebra.

The next theorem represents the most general form of the Lie-theoretical part of the solution of the restricted Burnside problem. It was announced by Zelmanov in [27]. A detailed proof can be found in [29].

Theorem 5 Let L be a Lie algebra over a field and suppose that L satisfies a PI. If L can be generated by a finite set $X$ such that every commutator in elements of $X$ is ad-nilpotent, then $L$ is nilpotent.

An important criterion for a Lie algebra to satisfy a PI is provided by the next theorem, which was proved by Bahturin and Zaicev for soluble groups of automorphisms [3] and later extended by Linchenko to the general case [13].

Theorem 6 Let L be a Lie algebra over a field $K$. Assume that a finite group A acts on $L$ by automorphisms in such a manner that $C_{L}(A)$ satisfies a PI. Assume further that the characteristic of $K$ is either 0 or prime to the order of A. Then L satisfies a PI.

We use the centralizer notation for the fixed-point subalgebra $C_{L}(A)$ of a group of automorphisms $A$ of $L$.

Another useful result, whose proof can be found in [10, Lemma 5], is the following.

Lemma 1 Let $L$ be a Lie algebra and $H$ a subalgebra of $L$ generated by $m$ elements $h_{1}, \ldots, h_{m}$ such that all commutators in the generators $h_{i}$ are ad-nilpotent in L. If $H$ is nilpotent, then we have $[L, \underbrace{H, \ldots, H}_{u}]=0$ for some number $u$.

Let $G$ be a (profinite) group. A series of subgroups

$$
G=G_{1} \geq G_{2} \geq \ldots
$$

is called an $N$-series if it satisfies $\left[G_{i}, G_{j}\right] \leq G_{i+j}$ for all $i, j \geq 1$. Here and throughout the paper, when dealing with a profinite group we consider only closed subgroups. Obviously any $N$-series is central, i.e. $G_{i} / G_{i+1} \leq Z\left(G / G_{i+1}\right)$ for any $i$. Let $p$ be a prime. An $N$-series is called $N_{p}$-series if $G_{i}^{p} \leq G_{p i}$ for all $i$. Given an $N$-series $(*)$, let $L^{*}(G)$ be the direct sum of the abelian groups $L_{i}^{*}=G_{i} / G_{i+1}$, written additively. Commutation in $G$ induces a binary operation [, ] in $L^{*}(G)$. For homogeneous elements $x G_{i+1} \in L_{i}^{*}, y G_{j+1} \in L_{j}^{*}$, the operation is defined by

$$
\left[x G_{i+1}, y G_{j+1}\right]=[x, y] G_{i+j+1} \in L_{i+j}^{*}
$$


and extended to arbitrary elements of $L^{*}(G)$ by linearity. It is easy to check that the operation is well defined and that $L^{*}(G)$ with the operations + and [, ] is a Lie ring. If all quotients $G_{i} / G_{i+1}$ of an $N$-series (*) have prime exponent $p$, then $L^{*}(G)$ can be viewed as a Lie algebra over $\mathbb{F}_{p}$, the field with $p$ elements. In the important case where the series $(*)$ is the $p$-dimensional central series (also known under the name of Zassenhaus-Jennings-Lazard series) of $G$, we write $D_{i}=D_{i}(G)$ for the $i$ th term of the series of $G, L(G)$ for the corresponding associated Lie algebra over the field with $p$ elements and $L_{p}(G)$ for the subalgebra generated by the first homogeneous component $D_{1} / D_{2}$ in $L(G)$. Observe that the $p$-dimensional central series is an $N_{p}$-series (see [8, p. 250] for details).

Any automorphism of $G$ induces naturally an automorphism of $L^{*}(G)$. If $G$ is profinite and $\alpha$ is a coprime automorphism of $G$, then the subalgebra of fixed points of $\alpha$ in $L^{*}(G)$ is isomorphic to the Lie algebra associated with the group $C_{G}(\alpha)$ via the series formed by intersections of $C_{G}(\alpha)$ with the terms of the series (*) (see [19] for more details).

Given an $N_{p}$-series $(*)$ of $G$, let $x \in G$ and let $i=i(x)$ be the largest positive integer such that $x \in G_{i}$. We denote by $x^{*}$ the element $x G_{i+1} \in L^{*}(G)$. We now quote some results providing sufficient conditions for $x^{*}$ to be ad-nilpotent. The first lemma is due to Lazard (see [11, p. 131]).

Lemma 2 For any $x \in G$, we have $\left(a d x^{*}\right)^{p}=a d\left(x^{p}\right)^{*}$. In particular, if $x$ is of finite order $t$, then $x^{*}$ is ad-nilpotent of index at most $t$.

The next result essentially is due to Wilson and Zelmanov since it follows from the proof of [25, Lemma in Section 3].

Lemma 3 Let $x$ be an Engel element of a profinite group $G$. Then $x^{*}$ is ad-nilpotent.

Combining Lemmas 2 and 3, it is easy to deduce the following result.

Lemma 4 Let $x$ be an element of a profinite group $G$ for which there exists a positive integer $d$ such that $x^{d}$ is Engel. Then $x^{*}$ is ad-nilpotent.

A group $G$ is said to satisfy a coset identity if there is a nontrivial group word $w=$ $w\left(x_{1}, x_{2}, \ldots, x_{k}\right)$ and cosets $g_{1} H, g_{2} H, \ldots, g_{k} H$ of a subgroup $H$ of $G$ of finite index such that $w\left(g_{1} h_{1}, g_{2} h_{2}, \ldots, g_{k} h_{k}\right)=1$ for all $h_{1}, h_{2}, \ldots, h_{k} \in H$; in this case, we can also say that the law $w \equiv 1$ is satisfied on the cosets $g_{1} H, g_{2} H, \ldots, g_{k} H$. In [25, Theorem 1], Wilson and Zelmanov proved the following theorem.

Theorem 7 If a profinite group $G$ has an open subgroup $H$ and elements $g_{1}, \ldots, g_{k}$ such that a law $w \equiv 1$ is satisfied on the cosets $a_{1} H, \ldots, a_{k} H$, then for each prime $p$ the Lie algebra $L_{p}(G)$ satisfies a $P I$.

\section{Proof of Theorem 3 and Corollary 1}

We start this section by proving the following useful result.

Lemma 5 Let p be a prime. Suppose that a finite group A acts coprimely on a profinite group $G$. Assume that some power of each element in $C_{G}(A)$ is Engel in $C_{G}(A)$ for any a $\in A^{\#}$. Then $L_{p}(G)$ satisfies a multilinear polynomial identity.

Proof Let $L=L_{p}(G)$. In view of Theorem 6, it is sufficient to show that $C_{L}(A)$ satisfies a polynomial identity. We know that $C_{L}(A)$ is isomorphic to the Lie algebra associated with 
the central series of $C_{G}(A)$ obtained by intersecting $C_{G}(A)$ with the $p$-dimensional central series of $G$. For each pair $i, j$ of positive integers, we set

$$
S_{i j}=\left\{(g, h) \in C_{G}(A) \times C_{G}(A):\left[g,{ }_{i} h^{j}\right]=1\right\} .
$$

Since the sets $S_{i j}$ are closed in $C_{G}(A) \times C_{G}(A)$ and their union is $C_{G}(A) \times C_{G}(A)$, by Baire's category theorem $[9$, p. 200] at least one of these sets has a nonempty interior. Therefore, we can find an open subgroup $H$ in $C_{G}(A)$, elements $u, v \in C_{G}(A)$ and integers $n, d$ such that the identity $\left[x,{ }_{n} y^{d}\right] \equiv 1$ is satisfied on the cosets $u H, v H$. Thus, Theorem 7 applies and $C_{L}(A)$ satisfies a polynomial identity, as desired.

Next, we will prove Theorem 3 under the additional hypothesis that $G$ is a pro- $p$ group.

Proposition 1 Let $G$ be a pro-p group satisfying the hypothesis of Theorem 3. Then $G$ is locally virtually nilpotent.

Proof Since every finite subset of $G$ is contained in a finitely generated $A$-invariant closed subgroup, we may assume that $G$ is finitely generated. Then, of course, it will be sufficient to show that $G$ is virtually nilpotent.

Let $H$ be the subgroup generated by all Engel elements in $G$. Note that $H$ is a normal $A$-invariant subgroup of $G$. Thus, for each $a \in A^{\#}$, we have $C_{G / H}(a)=C_{G}(a) H / H$, which is a torsion subgroup. By Theorem $1, G / H$ is finite, and so, $H$ is open. Since $G$ is finitely generated, [24, Proposition 4.3.1] implies that $H$ is finitely generated, as well. We claim that $H$ is nilpotent.

Indeed, we denote by $D_{j}=D_{j}(H)$ the terms of the $p$-dimensional central series of $H$. Let $L=L_{p}(H)$ be the Lie algebra associated with the group $H$ and $L_{j}=L \cap\left(D_{j} / D_{j+1}\right)$. Thus, $L=\oplus_{j \geq 1} L_{j}$. The group $A$ naturally acts on $L$. Let $A_{1}, \ldots, A_{q+1}$ be the distinct maximal subgroups of $A$. Set $L_{i j}=C_{L_{j}}\left(A_{i}\right)$. We know that any $A$-invariant subgroup is generated by the centralizers of $A_{i}$. Therefore, for any $j$ we have

$$
L_{j}=\sum_{i=1}^{q+1} L_{i j} .
$$

Further, for any $l \in L_{i j}$ there exists an element $x \in D_{j} \cap C_{H}\left(A_{i}\right)$ such that $l=x D_{j+1}$. Hence, there exists a positive integer $d$ such that $x^{d}$ is Engel in $H$. It follows from Lemma 4 that $l$ is ad-nilpotent in $L$. Thus,

any element in $L_{i j}$ is ad-nilpotent in $L$.

Let $\omega$ be a primitive $q$ th root of unity and $\bar{L}=L \otimes \mathbb{F}_{p}[\omega]$. Here $\mathbb{F}_{p}$ stands for the field with $p$ elements. We can view $\bar{L}$ both as a Lie algebra over $\mathbb{F}_{p}$ and as that over $\mathbb{F}_{p}[\omega]$. It is natural to identity $L$ with the subalgebra $L \otimes 1$ of $\bar{L}$. We note that if an element $x \in L$ is ad-nilpotent of index $r$, say, then the correspondent element $x \otimes 1$ is ad-nilpotent in $\bar{L}$ of the same index $r$.

Put $\bar{L}_{j}=L_{j} \otimes \mathbb{F}_{p}[\omega]$. Then $\bar{L}=\left\langle\bar{L}_{1}\right\rangle$ and $\bar{L}$ is the direct sum of the homogeneous components $\bar{L}_{j}$. The group $A$ naturally acts on $\bar{L}$, and we have $\bar{L}_{i j}=C_{\bar{L}_{j}}\left(A_{i}\right)$, where $\bar{L}_{i j}=L_{i j} \otimes \mathbb{F}_{p}[\omega]$. Let us show that

$$
\text { any element } y \in \bar{L}_{i j} \text { is ad-nilpotent in } \bar{L} \text {. }
$$

Since $\bar{L}_{i j}=L_{i j} \otimes \mathbb{F}_{p}[\omega]$, we can write

$$
y=x_{0}+\omega x_{1}+\omega^{2} x_{2} \cdots+\omega^{q-2} x_{q-2}
$$


for suitable $x_{0}, x_{1}, x_{2}, \ldots, x_{q-2} \in L_{i j}$. In view of (1), it is easy to see that each of the summands $\omega^{s} x_{s}$ is ad-nilpotent in $\bar{L}$. Let $J$ be the subalgebra of $\bar{L}$ generated by $x_{0}$, $\omega x_{1}, \omega^{2} x_{2}, \ldots, \omega^{q-2} x_{q-2}$. We wish to show that $J$ is nilpotent.

Note that $J \subseteq C_{\bar{L}}\left(A_{i}\right)$. A commutator of weight $t$ in the generators of $J$ has form $\omega^{\alpha} x$ for some $x$ that belongs to $L_{i m}$, where $m=t j$. By (1), the element $x$ is ad-nilpotent in $\bar{L}$, and so, such a commutator must be ad-nilpotent. By Lemma $5, L$ satisfies a multilinear polynomial identity. The multilinear identity is also satisfied in $\bar{L}$, and so, it is satisfied in $J$, since $J \subseteq C_{\bar{L}}\left(A_{i}\right)$. Hence, by Theorem $5 J$ is nilpotent. Now, applying Lemma 1 , we get that there exists some positive integer $u$ such that $[\bar{L}, \underbrace{J, \ldots, J}_{u}]=0$. This proves (2).

Since $A$ is abelian and the ground field is now a splitting field for $A$, every component $\bar{L}_{j}$ decomposes in the direct sum of common eigenspaces for $A$. In particular, $\bar{L}_{1}$ is spanned by finitely many common eigenvectors for $A$, since $H$ is a finitely generated pro- $p$ group. Hence, $\bar{L}$ is generated by finitely many common eigenvectors for $A$ from $\bar{L}_{1}$. Since $A$ is noncyclic, every common eigenvector is contained in the centralizer $C_{\bar{L}}\left(A_{i}\right)$ for some $i \leq q+1$.

We also note that any commutator in common eigenvectors is again a common eigenvector for $A$. Therefore, if $l_{1}, l_{2}, \ldots \in \bar{L}_{1}$ are common eigenvectors for $A$ generating $\bar{L}$, then any commutator in those generators belongs to some $\bar{L}_{i j}$ and so, by (2), is ad-nilpotent.

As we have mentioned earlier, $\bar{L}$ satisfies a polynomial identity. It follows from Theorem 5 that $\bar{L}$ is nilpotent. Since $L$ embeds into $\bar{L}$, we deduce that $L$ is nilpotent as well.

According to Lazard [12], the nilpotency of $L$ is equivalent to $H$ being $p$-adic analytic (for details see [12, A.1 in Appendices and Sections 3.1 and 3.4 in Ch. III] or [6, 1.(k) and 1.(o) in Interlude A]). It follows from [6, 7.19 Theorem] that $H$ admits a faithful linear representation over the field of $p$-adic numbers.

Since $H$ is a finitely generated pro- $p$ group and can be generated by Engel elements, by using an inverse limit argument combined with the Burnside basis theorem [17, 5.3.2], we see that $H$ is generated by finitely many Engel elements. A result of Gruenberg [7, Theorem 0] says that in a linear group the Hirsch-Plotkin radical coincides with the set of Engel elements. Then it follows that $H$ is nilpotent, as claimed. This concludes the proof.

As usual, for a profinite group $G$ we denote by $\pi(G)$ the set of prime divisors of the orders of finite continuous homomorphic images of $G$. We say that $G$ is a $\pi$-group if $\pi(G) \subseteq \pi$ and $G$ is a $\pi^{\prime}$-group if $\pi(G) \cap \pi=\emptyset$. If $m$ is an integer, we denote by $\pi(m)$ the set of prime divisors of $m$. If $\pi$ is a set of primes, we denote by $O_{\pi}(G)$ the maximal normal $\pi$-subgroup of $G$ and by $O_{\pi^{\prime}}(G)$ the maximal normal $\pi^{\prime}$-subgroup.

Now, we are ready to deal with the proof of Theorem 3.

Proof of Theorem 3 It will be convenient first to prove the theorem under the additional hypothesis that $G$ is pronilpotent. Therefore, $G$ is the Cartesian product of its Sylow subgroups. Choose $a \in A^{\#}$. For each pair $i, j$ of positive integers, we set

$$
S_{i j}=\left\{(x, y) \in G \times C_{G}(a):\left[x, i y^{j}\right]=1\right\} .
$$

Arguing as in the proof of Lemma 5, we deduce that there exist an open normal subgroup $H$ in $G$, elements $u \in G, v \in C_{G}(a)$ and positive integers $n, d$ such that $\left[u l,{ }_{n}(v k)^{d}\right]=1$ for any $l \in H$ and any $k \in H \cap C_{G}(a)$.

Let $[G: H]=m$ and let $\pi_{1}=\pi(m)$ be the set of primes dividing $m$. Denote $O_{\pi_{1}^{\prime}}(G)$ by $K$ and write $J$ for the Sylow subgroups of $G$ corresponding to the primes that belong to $\pi_{1}$. Since $G=J \times K=J H$, we deduce that $\left[x,{ }_{n} y^{d}\right]=1$, for all $x \in K$ and $y \in C_{K}(a)$. Now set $\pi_{2}=\pi(d)$ and $\pi=\pi_{1} \cup \pi_{2}$. Denote $O_{\pi^{\prime}}(G)$ by $T$. Since by construction $(p, d)=1$ for each prime $p \in \pi(T)$, it follows that every element $y$ in $C_{T}(a)$ is $n$-Engel in $T$. 
Of course, the set $\pi$ and the integer $n$ depend only on the choice of $a \in A^{\#}$, so strictly speaking they should be denoted by $\pi_{a}$ and $n_{a}$, respectively. We choose such $\pi_{a}$ and $n_{a}$ for

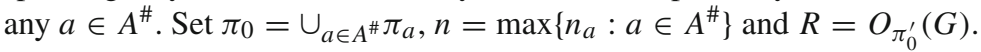

By construction, we see that, for each $a \in A^{\#}$, every element of $C_{R}(a)$ is $n$-Engel in $R$. Using an inverse limit argument, we deduce from [22, Theorem 1.2] that $R$ is $s$-Engel for some integer $s$. Thus, [25, Theorem 5] implies that $R$ is locally nilpotent. Let $p_{1}, \ldots, p_{r}$ be the finitely many primes in $\pi$ and $P_{1}, \ldots, P_{r}$ be the corresponding Sylow subgroups of $G$. Then $G=P_{1} \times \ldots \times P_{r} \times R$, and therefore, it is sufficient to show that each subgroup $P_{i}$ is locally virtually nilpotent. But, this is immediate from Proposition 1 . This proves the result in the particular case where $G$ is pronilpotent.

Let us now drop the assumption that $G$ is pronilpotent. Without loss of generality, we can assume that $G$ is finitely generated. Set $K$ be the closure of the subgroup of $G$ generated by all Engel elements in $G$. Note that $K$ is a normal $A$-invariant subgroup. Since $C_{G / K}(a)=$ $C_{G}(a) K / K$, for any $a \in A^{\#}$, in particular we know that each centralizer is torsion. Now Theorem 1 implies that $G / K$ is finite, and therefore, $K$ is finitely generated. By Baer's Theorem [17, 12.3.7], we deduce that $K$ is a pronilpotent group. Hence, using what we showed above, we conclude that $K$ is virtually nilpotent and this completes the proof.

We close this section by giving the proof of Corollary 1 .

Proof of Corollary 1 Suppose that the corollary is false. Then, for each pair of positive integers $i, j$, we can choose a group $G_{i j}$ satisfying the hypothesis of the corollary and having all of its normal subgroups either with nilpotency class at least $i$ or with index in $G_{i j}$ at least $j$ (or both properties). In each group $G_{i j}$, we fix generators $g_{1}^{i j}, \ldots, g_{m}^{i j}$.

Let $G$ be the Cartesian product of the groups $G_{i j}$, assuming that we use the lexicographic order to construct the Cartesian product. Note that $G$ is a profinite group admitting a coprime action of $A$ and such that all $d$ th power of elements in $C_{G}(a)$ are $n$-Engel in $G$ for each $a \in A^{\#}$. Thus, by Theorem $3, G$ is locally virtually nilpotent.

In $G$, consider the closed subgroup $D$ generated by $m$ elements

$$
g_{1}=\left(g_{1}^{11}, g_{1}^{12}, \ldots\right), \ldots, g_{m}=\left(g_{m}^{11}, g_{m}^{12}, \ldots\right) .
$$

Thus, $D$ has a open nilpotent normal subgroup $K$ of class $c$, say. Let $r$ be the index of $K$ in $D$ and observe that both $c$ and $r$ are numbers that depend only on $m, n, q$ and $d$. We remark that each of the groups $G_{i j}$ is isomorphic to a finite quotient of $D$. Thus, each subgroup $G_{i j}^{r}$ is nilpotent of class at most $c$. Furthermore, by the positive solution of the restricted Burnside problem [26,27,29], we know the index of $G_{i j}^{r}$ in $G_{i j}$ depends only on $m$ and $r$. This leads to a contradiction.

\section{Proof of Theorem 4 and Corollary 2}

Let $F$ denote the free group on free generators $x_{1}, x_{2}, \ldots$ Recall that a positive word in $X=\left\{x_{1}, x_{2}, \ldots\right\}$ is any nontrivial element of $F$ not involving the inverses of the $x_{i}$. A positive (or semigroup) law of a group $G$ is a nontrivial identity of the form $u \equiv v$ where $u, v$ are positive words in $F$, holding under every substitution of elements of $X$ by elements of $G$. The maximum of lengths of $u$ and $v$ is called the degree of the law $u \equiv v$.

By a result of Mal'cev [15] (see also [16]), a group that is an extension of a nilpotent group by a group of finite exponent satisfies a positive law. More precisely, Mal'cev discovered a positive law $M_{c}(x, y)$ in two variables and of degree $2^{c}$ that holds in any nilpotent group of 
class $c$. Therefore, if $G$ is an extension of a nilpotent group of class $c$ by a group of exponent $e$, then $G$ satisfies the positive law $M_{c}\left(x^{e}, y^{e}\right)$. The explicit form of the Mal'cev law will not be required in this paper.

Next result is a profinite version of [21, Theorem A].

Lemma 6 Let $q$ be a prime and A an elementary abelian group of order $q^{3}$. Suppose that $A$ acts coprimely on a profinite group $G$ and assume that $C_{G}(a)$ satisfies a positive law of degree $n$ for each $a \in A^{\#}$. Then $G$ satisfies a positive law as well.

Proof The result follows easily by using an inverse limit argument and noting that, by the proof of [21, Theorem A], any finite quotient of $G$ over an $A$-invariant open normal subgroup $N$ satisfies the positive law $M_{c}\left(x^{k}, y^{k}\right)$, for some positive integers $c$ and $k$ which do not depend on the choice of $N$ but only on $n$ and $q$.

We are ready to embark on the proof of Theorem 4. First we consider the case where $G$ is a pro- $p$ group.

Proposition 2 Let $G$ be a pro-p group satisfying the hypothesis of Theorem 4. Then $G$ is locally virtually nilpotent.

Proof Since every finite set of $G$ is contained in a finitely generated $A$-invariant closed subgroup, we may assume that $G$ is finitely generated. It will be sufficient to show that $G$ is virtually nilpotent.

We denote by $D_{j}=D_{j}(G)$ the terms of the $p$-dimensional central series of $G$. Let $L=L_{p}(G)$ be the Lie algebra associated with the group $G$ and $L_{j}=L \cap\left(D_{j} / D_{j+1}\right)$. Thus, $L=\oplus_{j \geq 1} L_{j}$. The group $A$ naturally acts on $L$. Let $A_{1}, \ldots, A_{s}$ be the distinct maximal subgroups of $A$. Since each subgroup $A_{i}$ is noncyclic we get $L=\sum_{a \in A_{i}} C_{L}(a)$, for every $i \leq s$. Set $L_{i j}=C_{L_{j}}\left(A_{i}\right)$. Hence, for any $j$ we get

$$
L_{j}=\sum_{i=1}^{s} L_{i j} .
$$

Thus, for any $l \in L_{i j}$ there exists an element $x \in D_{j} \cap C_{G}\left(A_{i}\right)$ such that $l=x D_{j+1}$. By assumption, some power of $x$ is Engel in $C_{G}\left(A_{i}\right) \subseteq C_{G}(a)$, for some $a \in A_{i}$. It follows from Lemma 4 that $l$ is ad-nilpotent in $C_{L}(a)$ for every $a \in A_{i}^{\#}$. Since $L=\sum_{a \in A_{i}} C_{L}(a)$, we deduce that any element $l \in L_{i j}$ is ad-nilpotent in $L$. Now, mimicking the argument that we used in the proof of Proposition 1, with only obvious changes, one can show that $L$ is nilpotent. We omit further details.

According to Lazard [12], the nilpotency of $L$ is equivalent to $G$ being $p$-adic analytic. The Lubotzky-Mann theory [14] ensures that $G$ has finite rank. Then, each centralizer $C_{G}(a)$ is finitely generated. Now, applying [4, Theorem 1.1], we know that all centralizers $C_{G}(a)$ are virtually nilpotent. Thus, there exist a $p$-power $k$ and a positive integer $c$ such that, for each $a \in A^{\#}$, the subgroup $C_{G}(a)^{k}$ has nilpotency class at most $c$. A result of Mal'cev [15] implies now that all centralizers $C_{G}(a)$ satisfy the positive law $M_{c}\left(x^{k}, y^{k}\right)$, and so, Lemma 6 yields that $G$ satisfies a positive law too. In accordance with the theorem by Burns, Macedońska and Medvedev [5], the group $G$ is an extension of a nilpotent group $N$ by a group of finite exponent. Finally, it follows from [28, Theorem 1] that $G / N$ is finite and this completes the proof.

Recall that the Fitting subgroup of a finite group $H$ is the unique largest normal nilpotent subgroup of $H$, which will be denoted by $F(H)$. Similarly, for any profinite group $G$, we will 
denote by $F(G)$ the (unique) largest normal pronilpotent subgroup of $G$. We remark that any Engel element in a profinite group $G$ belongs to $F(G)$. Indeed, let $K$ be the closed subgroup of $G$ generated by the set of all Engel elements in $G$. By Baer's theorem [17, 12.3.7], the image of $K$ in every finite quotient of $G$ is nilpotent. Since $K$ is normal in $G$, we see that $K$ is pronilpotent and so, in particular, contained in $F(G)$.

Proof of Theorem 4 Let $A_{1}, \ldots, A_{s}$ be the distinct maximal subgroups of $A$. Fix $A_{i}$ and take $x \in C_{G}\left(A_{i}\right)$. Note that $C_{G}\left(A_{i}\right) \subseteq C_{G}(a)$ for any $a \in A_{i}$. So, by assumption, there exists a positive integer $u_{a}$, depending on $a$, such that $x^{u_{a}} \in F\left(C_{G}(a)\right)$. Then there exist positive integers $u_{1}, \ldots, u_{q^{2}}$ such that $x^{u_{1} \cdots u_{q^{2}}} \in F\left(C_{G}(a)\right)$, for all $a \in A_{i}$.

Let $N$ be any $A$-invariant open normal subgroup of $G$. By [21, Lemma 2.6], we know that the image of $x^{u_{1} \ldots u_{q}}$ in the finite quotient $G / N$ belongs to $\bigcap_{a \in A_{i}^{\#}} F\left(C_{G / N}(a)\right) \leq F(G / N)$. Thus, the element $x^{u_{1} \ldots u_{q^{2}}}$ belongs to $F(G)$. Since $A_{i}$ and $x$ were chosen arbitrarily, we can repeat the argument for any $x \in C_{G}\left(A_{i}\right)$ and $i \in\{1, \ldots, s\}$. Then the images of the centralizers $C_{G}\left(A_{i}\right)$ in the quotient group $\bar{G}=G / F(G)$ are all torsion subgroups.

Let $a \in A^{\#}$ and consider $K=C_{\bar{G}}(a)$. The group $A$ naturally acts on $K$ inducing an automorphism group $A_{0}$. Further, for any $\alpha \in A_{0}$ the centralizer $C_{K}(\alpha)$ is exactly $C_{\bar{G}}\left(A_{i}\right)$, where $A_{i}=\left\langle a, a_{1}\right\rangle$ and $a_{1}$ is the element of $A$ that induces $\alpha$ (in the action of $A$ on $K$ ). We claim that $C_{K}(\alpha)$ is torsion, for every $\alpha \in A_{0}$. Indeed, if $A_{0}$ has order $q^{2}$, then it follows from Theorem 1 that $K$ is torsion. If the order of $A_{0}$ is less than $q^{2}$, then $K=C_{\bar{G}}(a) \leq C_{\bar{G}}\left(A_{i}\right)$, and so, $K$ is torsion as well. Thus, we get that $C_{\bar{G}}(a)$ is torsion for any $a \in A^{\#}$. Applying again Theorem 1, we deduce that $\bar{G}=G / F(G)$ is torsion and, in particular, locally finite.

The above argument shows that it is enough to prove the theorem under the additional assumption that $G$ is pronilpotent. Choose now $a \in A^{\#}$. For each pair $i, j$ of positive integers, we set

$$
S_{i j}=\left\{(x, y) \in C_{G}(a) \times C_{G}(a):\left[x, i y^{j}\right]=1\right\} .
$$

With an argument similar to that used in the proof of Theorem 3, with only obvious changes, we can show that $G=P_{1} \times \ldots \times P_{r} \times R$, where $R$ is a locally nilpotent subgroup of $G$ and $P_{i}$ are finitely many Sylow subgroups of $G$. Therefore, it is sufficient to show that each subgroup $P_{i}$ is locally virtually nilpotent. This follows from Proposition 2 and the proof is complete.

We conclude observing that the proof of Corollary 2 is analogous to that of Corollary 1 and can be obtained, with obvious changes, by replacing every appeal to Theorem 3 in the proof of Corollary 1 by an appeal to Theorem 4 . Therefore, we omit the full details.

\section{References}

1. Acciarri, C., Shumyatsky, P.: Profinite groups and the fixed points of coprime automorphisms. J. Algebra 452, 188-195 (2016)

2. Acciarri, C., Shumyatsky, P., Silveira, D.S.: On groups with automorphisms whose fixed points are Engel. Ann. Mater. Pura Appl. 197, 307-316 (2018)

3. Bahturin, Y.A., Zaicev, M.V.: Identities of graded algebras. J. Algebra 205, 1-12 (1998)

4. Bastos, R., Shumyatsky, P.: On profinite groups with Engel-like conditions. J. Algebra 427, 215-225 (2015)

5. Burns, R.G., Macedonska, O., Medvedev, Yu.: Groups satisfying semigroup laws, and nilpotent-byBurnside varieties. J. Algebra 195, 510-525 (1997)

6. Dixon, J.D., du Sautoy, M.P.F., Mann, A., Segal, D.: Analytic Pro-p Groups. Cambridge University Press, Cambridge (1991) 
7. Gruenberg, K.W.: The Engel structure of linear groups. J. Algebra 3, 291-303 (1966)

8. Huppert, B., Blackburn, N.: Finite Groups II. Springer, Berlin (1982)

9. Kelly, J.L.: General Topology. Van Nostrand, Toronto (1955)

10. Khukhro, E.I., Shumyatsky, P.: Bounding the exponent of a finite group with automorphisms. J. Algebra 212, 363-374 (1999)

11. Lazard, M.: Sur les groupes nilpotents et les anneaux de Lie. Ann. Sci. Éc. Norm. Supér. 71, 101-190 (1954)

12. Lazard, M.: Groupes analytiques $p$-adiques. Publ. Math. Inst. Hautes Études Sci. 26, 389-603 (1965)

13. Linchenko, V.: Identities of Lie algebras with actions of Hopf algebras. Comm. Algebra 25, 3179-3187 (1997)

14. Lubotzky, A., Mann, A.: Powerful p-groups. I, II. J. Algebra 105, 484-515 (1987)

15. Mal'cev, A .I.: Nilpotent semigroups. Ivanov. Gos. Ped. Inst. Uč. Zap. Fiz. Mat. Nauki 4, 107-111 (1953)

16. Neumann, B.H., Taylor, T.: Subsemigroups of nilpotent groups. Proc. R. Soc. Lond. Ser. A 274, 1-4 (1963)

17. Robinson, D.J.S.: A Course in the Theory of Groups. Springer, New York (1996)

18. Shumyatsky, P.: Centralizers in groups with finiteness conditions. J. Group Theory 1, 275-282 (1998)

19. Shumyatsky, P.: Applications of Lie ring methods to group theory. In: Costa, R., et al. (Eds.) Nonassociative Algebra and Its Applications, pp. 373-395. Marcel Dekker, New York (2000)

20. Shumyatsky, P.: Coprime automorphisms of profinite groups. Q. J. Math. 53, 371-376 (2002)

21. Shumyatsky, P.: Positive laws in fixed points. Trans. Am. Math. Soc. 356, 2081-2091 (2004)

22. Shumyatsky, P., Silveira, D.S.: On finite groups with automorphisms whose fixed points are Engel. Arch. Math. 106, 209-218 (2016)

23. Wilson, J.S.: On the structure of compact torsion groups. Monatsh. Math. 96, 57-66 (1983)

24. Wilson, J.S.: Profinite Groups. Clarendon Press, Oxford (1998)

25. Wilson, J.S., Zelmanov, E.I.: Identities for Lie algebras of pro- $p$ groups. J. Pure Appl. Algebra 81, 103-109 (1992)

26. Zelmanov, E.I.: Lie Methods in the Theory of Nilpotent Groups, in Groups '93 Galaway/ St Andrews, pp. 567-585. Cambridge University Press, Cambridge (1995)

27. Zelmanov, E.I.: Nil Rings and Periodic Groups. The Korean Math. Soc. Lecture Notes in Math, Seoul (1992)

28. Zelmanov, E.I.: On periodic compact groups. Isr. J. Math. 77, 83-95 (1992)

29. Zelmanov, E.I.: Lie algebras and torsion groups with identity. J. Comb. Algebra 1, 289-340 (2017)

Publisher's Note Springer Nature remains neutral with regard to jurisdictional claims in published maps and institutional affiliations. 